\title{
Radiologie als Wegbereiter für die „Active Surveillance“
}

Interview mit PD Dr. Dirk Beyersdorff, Diagnostische Radiologie - Universitätsklinikum Hamburg-Eppendorf, zur Aktualisierung der interdisziplinären Leitlinie der Qualität S3 zur Früherkennung, Diagnose und Therapie der verschiedenen Stadien des Prostatakarzinoms.

Herr Dr. Beyersdorff, 2009 wurde die S3-Leitlinie zur Früherkennung, Diagnose und Therapie der verschiedenen Stadien des Prostatakarzinoms erstmalig veröffentlicht. Sie waren damals wie heute an der mittlerweile dritten Aktualisierung beteiligt. Welche Rolle spielen Radiologen im Kampf gegen das Prostatakarzinom?
Bildgebende Verfahren der Radiologie haben in mehreren Phasen einen großen Einfluss auf die Behandlung des Prostatakarzinoms: beim Tumornachweis, beim Staging eines nachgewiesenen Karzinoms und im Verlauf einer Behandlung. In zahlreichen Studien konnte der Stellenwert der MRT für den Tumornachweis gezeigt werden. In den letzten Jahren hat der Anteil der MRT-gesteuerten Biopsien, seien es nun „in-bore“Biopsien oder Fusionsbiopsien, stark zugenommen, was die wachsende klinische Bedeutung für den Nachweis des Prostatakarzinoms widerspiegelt. Vor einer nerverhaltenden Operationstechnik, die heutzutage die Regel darstellt, ist ein präzises Staging in der Vorbereitung hilfreich, um

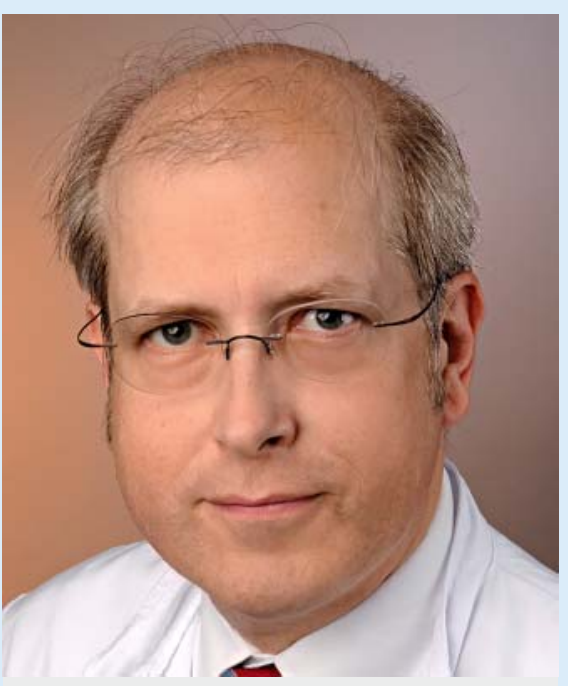

PD Dr. Dirk Beyersdorff 
eine individuelle Aufklärung zu ermöglichen. Wenn z. B. ein Tumor ein neurovaskuläres Bündel infiltriert, ist eine Nerverhaltung auf dieser Seite nicht angebracht.

Die Aktualisierung der Leitlinie erfolgt modular als "living guideline“ in einbis zweijährigen Abständen. Welche Neuerungen beinhaltet die aktuelle Version 4.0?

In der aktuellen Leitlinie wurde die Möglichkeit einer gezielten Biopsie von Befunden aus der Bildgebung aufgenommen. Wohl insbesondere auf Wunsch der überproportional vertretenen urologischen Experten wurde insbesondere die MRT-gesteuerte Fusionsbiopsie als eine Möglichkeit der gezielten Biopsie aufgenommen. Eine nur gezielte Biopsie, also ohne systematische Abdeckung der gesamten Prostata, ist weiterhin nicht vorgesehen. Zusätzlich wurde die Möglichkeit einer PET Hybrid-Bildgebung mit radioaktiv markierten PSMA-Liganden für die Rezidivdiagnostik in die Leitlinie aufgenommen.

$\mathrm{Zu}$ den bestehenden diagnostischen Möglichkeiten ist die MRT-UltraschallFusionsbiopsie hinzugekommen, allerdings „nur“ als offene Empfehlung. Warum ist das so?

Diese Empfehlung ist aus meiner Sicht nur als Zwischenschritt zu bewerten. Die Radiologie ist mit ihren Vertretern nur eine kleine Gruppe innerhalb des Leitlinienteams. Als Radiologen können wir das Potenzial unserer Techniken früh einschätzen und sehen hier anhand erster Studienergebnisse klinische Einsätze schon lange, bevor es der Mehrheit der anderen Leitlinienpartner möglich ist. Häufig sind ein langer Überzeugungsweg und ein langwieriges Abwarten auf Studienergebnisse mit dann stärkerer
Evidenz nötig, um eingetretene Behandlungspfade in Frage zu stellen. Manchmal können auch berufspolitische Interessen der Fachgesellschaften diese Entwicklung zusätzlich verzögern.

Ist die Radiologie eigentlich auch in die noch ausstehende Aktualisierung der Patientenleitlinie eingebunden?

Die Patientenleitlinie ist eine zusammenfassende und für einen medizinischen Laien verständliche Version der Leitlinie. Die Empfehlungen in diesem Ratgeber sollten nicht von denen der konsentierten Leitlinie abweichen. Diese aufwändige und eher redaktionelle Arbeit wird nur von einem Teil des Leitlinienteams gemacht. Die Radiologie ist hier nicht vertreten.

Nach der Aktualisierung ist vor der Aktualisierung. Version $\mathbf{4 . 0}$ ist bis zum 30.09.2017 gültig, die Leitlinie wurde bereits wieder als Leitlinienvorhaben angemeldet. Ein für die Leitlinienarbeit ganz normaler Vorgang oder leitet sich aus dem spezifischen Thema eine vergleichsweise kurzfristige Aktualisierungsnotwendigkeit $a b$ ?

Aus meiner Sicht besteht bezüglich der Bildgebung ein erheblicher Aktualisierungsbedarf, der bei der letzten Aktualisierung nicht berücksichtigt wurde. Das Steuerungsgremium, in dem die Radiologie nicht vertreten ist, hatte bei der letzten Aktualisierung von vorneherein nur einige Punkte aufgenommen. Die Radiologie ist aber für die anstehende Leitlinienaktualisierung im Vorfeld eingebunden. In der Leitliniengruppe sind neben der AG Uroradiologie und Urogenitaldiagnostik auch die AG Onkologische Bildgebung und der Berufsverband der Deutschen Radiologen vertreten. Die Indikation für die multiparametrische MRT der Prostata muss meiner Meinung nach vollständig überarbeitet werden und einen breiten Eingang in die Leitlinie finden. Derzeit wird dafür die Rolle der MRT in Form von systematischen Literaturrecherchen analysiert. Als Voraussetzung für die Aufnahme in die Leitlinie wurde von einer Arbeitsgruppe der AG Uroradiologie und Urogenitaldiagnostik zunächst eine Empfehlung zur Vorbereitung und Durchführung der multiparametrischen ProstataMRT erarbeitet (Franiel et al. RöFo 2016).

Das Prostatakarzinom ist in Deutschland die häufigste Krebserkrankung bei Männern - Tendenz steigend. Wie schätzen Sie die weitere Entwicklung in der medizinischen Versorgung ein und welche Perspektiven prognostizieren Sie mit Blick auf die Radiologie?

Im Laufe der letzten Jahre hat sich durch das Internet die Möglichkeit, Informationen zu einer Erkrankung und damit auch zu den diagnostischen Möglichkeiten zu erhalten, deutlich verbessert. Viele Männer mit einem erhöhten PSA-Wert wünschen deshalb von sich aus ein multiparametrisches MRT vor der invasiven Biopsie. Die Folge ist nicht nur eine Zunahme der Prostata-MRT, sondern damit auch eine Zunahme von gezielten, MRT-gesteuerten Biopsien. Dies wiederum sollte zu repräsentativeren Biopsie-Ergebnissen führen. Je besser uns die prätherapeutische Charakterisierung eines Prostatakarzinoms gelingt, umso sicherer ist eine Behandlungsform, bei der nicht die gesamte Prostata entfernt und histologisch aufgearbeitet wird. Ich sehe uns deshalb als Wegbereiter für die „Active Surveillance“ und die fokale Therapie mit multiparametrischem MRT der Prostata in der Vorbereitung und im Verlauf. Ob wir tatsächlich auch eine Rolle bei der bildgesteuerten fokalen Therapie spielen werden, ist derzeit noch offen. 